\title{
Integral transform solution for hyperbolic heat conduction in a finite slab
}

\author{
Evaldiney R. Monteiro ${ }^{a}$, Emanuel N. Macêdo ${ }^{b}$, João N.N. Quaresma ${ }^{\text {b,* }}$, Renato M. Cotta ${ }^{c}$ \\ a Mining Engineering Department, CSSPA, Campus de Marabá, Universidade Federal do Pará, UFPA \\ b School of Chemical Engineering, FEQ/ITEC/UFPA, Universidade Federal do Pará, 66075-110, Belém, PA, Brazil \\ c Mechanical Engineering Department - POLI E' COPPE, Universidade Federal do Rio de Janeiro, UFRJ, Cx. Postal 68503 - Cidade Universitária, 21945-970, Rio de Janeiro, RJ, Brazil
}

\section{A R T I C L E I N F O}

Available online 14 February 2009

\section{Keywords:}

Hyperbolic heat conduction

Generalized integral transform technique

Laplace transform

Finite volume-Gear method

\begin{abstract}
A B S T R A C T
An analytical integral transformation of the thermal wave propagation problem in a finite slab is obtained through the generalized integral transform technique (GITT). The use of the GITT approach in the analysis of the hyperbolic heat conduction equation leads to a coupled system of second order ordinary differential equations in the time variable. The resulting transformed ODE system is then numerically solved by Gear's method for stiff initial value problems. Numerical results are presented for the local and average temperatures with different Biot numbers and dimensionless thermal relaxation times, permitting a critical evaluation of the technique performance. A comparison is also performed with previously reported results in the literature for special cases and with those produced through the application of the Laplace transform method (LTM), and the finite volume-Gear method (FVGM).
\end{abstract}

(c) 2009 Elsevier Ltd. All rights reserved.

\section{Introduction}

Hyperbolic heat conduction is found in several engineering applications related to ultra-rapid time varying heat sources or contacts, such as with laser and microwave sources with extremely short duration or very high frequency, and more recently in association with nano-scale heat transfer in dispersed phase systems of nanoparticles.

The classical theory of heat conduction based on Fourier's law is not adequate to describe the physical mechanism of heat propagation for such situations, since the classical constitutive relation leads to paradoxical results in problems dealing with high rates of temperature changes. To overcome this limitation, Vernotte [1] and Cattaneo [2], based on the concept of heat transmission by waves, independently introduced an improvement of Fourier's law to describe problems involving high rates of temperature change, heat flow in an extremely short period of time or very low temperatures near absolute zero. Afterwards, a number of research contributions have been dedicated to the study of problems involving hyperbolic heat conduction [3-19].

More recently, Duhamel [19] provided a thorough review on the subject and proposed a finite integral transform pair for hyperbolic heat conduction problems in heterogeneous media. In this same context and with a complementary scope, the goal of the present work is to propose a more flexible analytical solution for the thermal wave propagation problem in a finite slab through the generalized integral transform technique (GITT) [20-28] with a local-instantaneous

\footnotetext{
is Communicated by W.J. Minkowycz.

* Corresponding author.

E-mail address: quaresma@ufpa.br (J.N.N. Quaresma).
}

filtering strategy $[29,30]$. The use of this integral transform approach in solving the hyperbolic heat conduction equation leads to a coupled system of second order ordinary differential equations in the time variable, which is then handled by the related algebraic eigenvalue problem or numerically solved by Gear's method for stiff initial value problems, such as available in subroutine DIVPAG of the IMSL Library [31]. Numerical results are here presented for the local and average temperatures with different values of the governing parameters, i.e., Biot numbers and dimensionless thermal relaxation times, allowing for a critical evaluation of the eigenfunction expansions behavior. A comparison with previously reported results for special cases is also performed. Finally, a couple of alternative approaches are applied to the same problem formulation for comparison purposes, namely a solution developed via the Laplace transform method (LTM) with numerical inversion, as well as a method of lines variation based on application of the finite volume method in the spatial coordinate and Gear's method in the time variable (FVGM).

\section{Mathematical formulation}

The classical theory of heat conduction based on the constitutive Fourier's law relates the heat flux density vector $\mathbf{q}(\mathbf{x}, \mathrm{t})$ to the temperature gradient $\nabla T(\mathbf{x}, t)$, while assuming that heat propagates with an infinite speed within the conducting medium, in accordance with:

$$
\mathrm{q}(\mathbf{x}, t)=-k \nabla T(\mathbf{x}, t)
$$

A modified form of Eq. (1) was independently and originally presented by Vernotte [1] and Cattaneo [2], for the case when the 


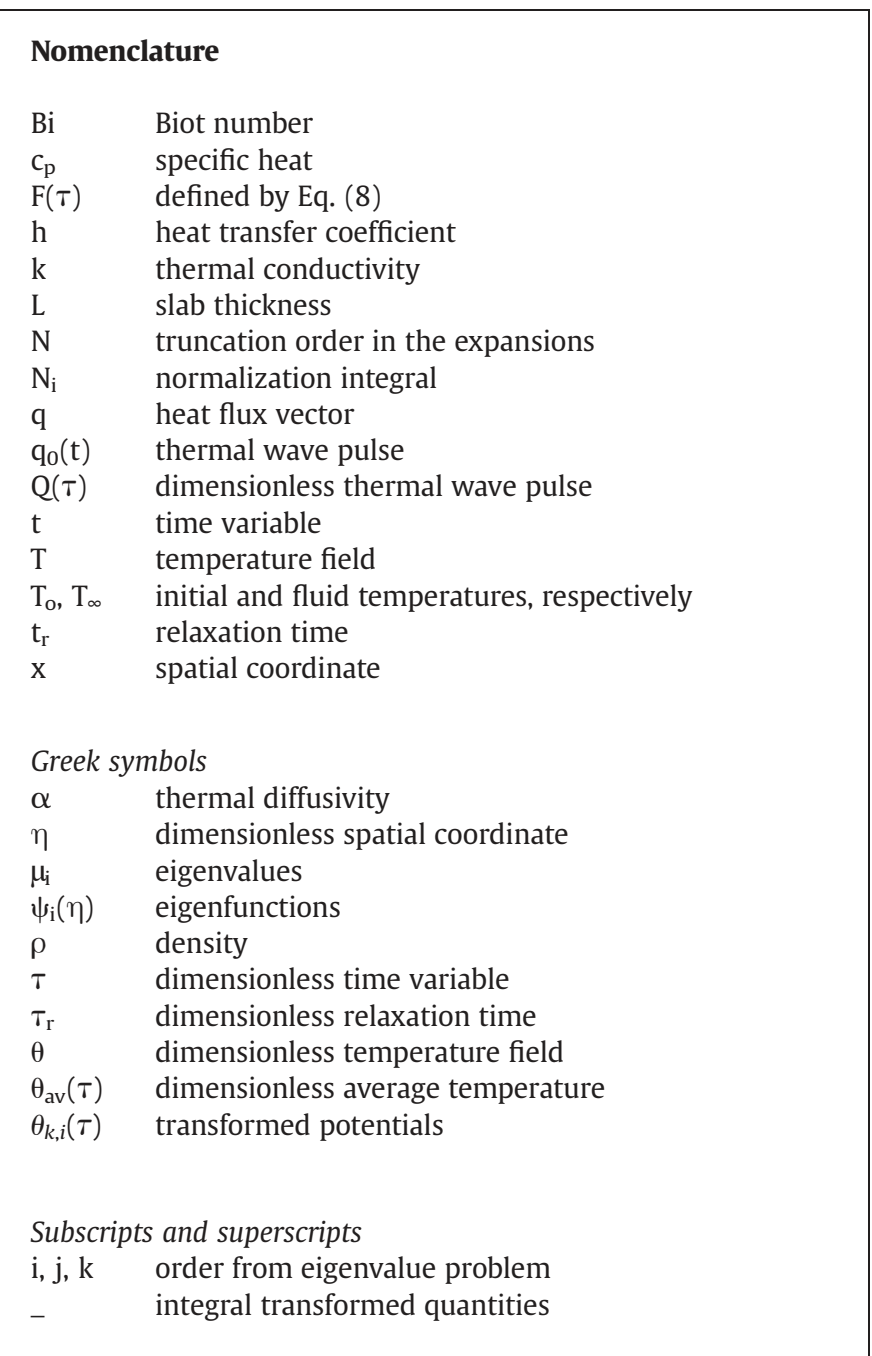

time scale of the local temperature variation is very small, and by considering a finite speed of wave propagation, resulting in:

$\mathrm{q}\left(\mathbf{x}, t+t_{r}\right)=-k \nabla T(\mathbf{x}, t)$

where $t_{\mathrm{r}}$, the so-called thermal relaxation time, is a thermodynamic property of the material where heat conduction occurs. However, the local character of the heat conduction theory is restored by a truncation of Eq. (2) to a first order expansion in $t_{\mathrm{r}}$ [1,2,12], namely:

$\mathbf{q}(\mathbf{x}, t)+t_{r} \frac{\partial \mathbf{q}(\mathbf{x}, t)}{\partial t}=-k \nabla T(\mathbf{x}, t)$

The energy equation in terms of the heat flux is then written as

$\rho c_{p} \frac{\partial T(\mathbf{x}, t)}{\partial t}=-\nabla \cdot \mathbf{q}(\mathbf{x}, t)+g(\mathbf{x}, t)$

One then combines Eq. (3) with the energy Eq. (4) and, assuming constant physical properties, recovers the hyperbolic heat conduction equation:

$t_{r} \frac{\partial^{2} T(\mathbf{x}, t)}{\partial t^{2}}+\frac{\partial T(\mathbf{x}, t)}{\partial t}=\alpha \nabla^{2} T(\mathbf{x}, t)+\frac{g(\mathbf{x}, t)}{\rho c_{p}}+\frac{t_{r}}{\rho c_{p}} \frac{\partial g(\mathbf{x}, t)}{\partial t}$

Eq. (5) models the temperature distribution for heat propagation in solids with a finite speed $\nu=\left(\alpha / t_{\mathrm{r}}\right)^{1 / 2}$, where $\alpha$ is the thermal diffusivity.
For illustration of the proposed approach, we here consider a test case of hyperbolic heat conduction in a slab of thickness $L$, initially at the uniform temperature $T_{0}$, subject to a prescribed heat flux at the boundary $\mathbf{x}=0$ and exchanging heat by convection at $\mathbf{x}=\mathrm{L}$ with a heat transfer coefficient, $h$, and a fluid with a constant temperature, $T_{\infty}$. Assuming constant thermophysical properties, $k$ and $\alpha$, and no internal heat generation, the unsteady one-dimensional formulation for this problem in dimensionless form is then written as:

$$
\begin{aligned}
& \frac{\partial \theta}{\partial \tau}+\tau_{r} \frac{\partial^{2} \theta}{\partial \tau^{2}}=\frac{\partial^{2} \theta}{\partial \eta^{2}}, \quad 0<\eta<1, \quad \tau>0 \\
& \theta(\eta, 0)=1 ; \quad \frac{\partial \theta(\eta, 0)}{\partial \tau}=0, \quad 0 \leq \eta \leq 1 \\
& \frac{\partial \theta(0, \tau)}{\partial \eta}=-\mathrm{F}(\tau) ; \quad \frac{\partial \theta(1, \tau)}{\partial \eta}+\operatorname{Bi} \theta(1, \tau)+\operatorname{Bi} \tau_{r} \frac{\partial \theta(1, \tau)}{\partial \tau}=0, \quad \tau>0
\end{aligned}
$$

where the various dimensionless groups are given by:

$$
\begin{gathered}
\theta(\eta, \tau)=\frac{T(\mathrm{x}, t)-T_{\infty}}{T_{0}-T_{\infty}} ; \quad \eta=\frac{\mathrm{x}}{L} ; \quad \tau=\frac{\alpha t}{L^{2}} ; \quad \mathrm{Bi}=\frac{\mathrm{hL}}{k} ; \quad \tau_{r}=\frac{\alpha \mathrm{t}_{r}}{L^{2}} ; \\
Q(\tau)=\frac{\mathrm{Lq}_{0}(t)}{k\left(T_{0}-T_{\infty}\right)}
\end{gathered}
$$

and the function $F(\tau)$ in the boundary source term of Eq. (6d) becomes

$F(\tau)=Q(\tau)+\tau_{r} Q^{\prime}(\tau)$

The following analysis can be performed for any type of wave pulses, but square and triangular pulses were here chosen to illustrate the solution behavior, which are described by the following functions (Fig. 1), respectively:

$Q(\tau)=\left\{\begin{array}{ll}0 ; \text { for } 0 \leq \tau<1 \\ 1 ; \text { for } 1 \leq \tau \leq 2 ; \\ 0 ; \text { for } \tau>2\end{array} \quad Q(\tau)=\left\{\begin{array}{cc}0 ; & \text { for } 0 \leq \tau<1 \\ \tau+\tau_{r}-1 ; & \text { for } 1 \leq \tau \leq 2 \\ 0 ; & \text { for } \tau>2\end{array}\right.\right.$

\section{Solution methodology}

The first step in application of the generalized integral transform technique (GITT) is the proposition of a filtering solution, which reduces the adverse effects on convergence rates due to the boundary source terms. The employed procedure is the adoption of a local-instantaneous analytical filter $[29,30]$, here applied to chosen stages in time of the original potential as:

$\theta(\eta, \tau)=\theta_{f, k}(\eta ; \tau)+\theta_{p, k}(\eta, \tau), \quad \tau_{k-1}<\tau<\tau_{k}$

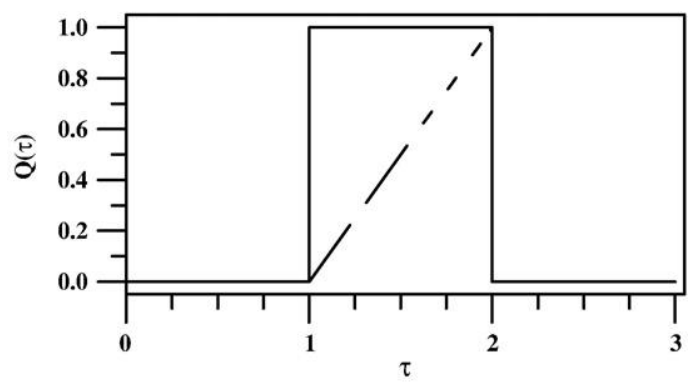

Fig. 1. Wave pulses for $Q(\tau)$ (square pulse, solid line, and triangular pulse, dashed line). 
The quasi-steady filter problem formulation is chosen in implicit form as:

$\frac{\partial^{2} \theta_{f, k}}{\partial \eta^{2}}=0, \quad 0<\eta<1, \quad \tau_{k-1}<\tau<\tau_{k}$

$\frac{\partial \theta_{f, k}(0, \tau)}{\partial \eta}=-F(\tau) \quad \frac{\partial \theta_{f, k}(1, \tau)}{\partial \eta}+B i \theta_{f, k}(1, \tau)=\gamma_{k-1}$

where the boundary condition transient term that appears in the filter is written as

$\gamma(\tau)=-B i \tau_{r} \frac{\partial \theta(1, \tau)}{\partial \tau} ; \quad \gamma_{k-1}=\gamma\left(\tau_{k-1}\right)$

The solution of Eq. (11) is readily obtained as

$\theta_{f, k}(\eta ; \tau)=F(\tau)(1-\eta)+\left[F(\tau)+\gamma_{k-1}\right] / B i$

The resulting problem formulation for the filtered potential, $\theta_{\mathrm{p}, \mathrm{k}}(\eta$, $\tau)$, is thus given by:

$\frac{\partial \theta_{p, k}}{\partial \tau}+\tau_{r} \frac{\partial^{2} \theta_{p, k}}{\partial \tau^{2}}=\frac{\partial^{2} \theta_{p, k}}{\partial \eta^{2}}+G_{k}(\eta, \tau), \quad 0<\eta<1, \quad \tau_{k-1}<\tau<\tau_{k}$

$\theta_{p, k}\left(\eta, \tau_{k-1}\right)=\left\{\begin{array}{ll}1-\theta_{f, k}, & \text { for } k=1 \\ \theta_{p, k-1}+\theta_{f, k-1}-\theta_{f, k}, & \text { for } k>1\end{array}, \quad 0 \leq \eta \leq 1\right.$

$\frac{\partial \theta_{p, k}\left(\eta, \tau_{k-1}\right)}{\partial \tau}=\left\{\begin{array}{ll}\frac{\partial \theta_{f, k}}{\partial \tau}, & \text { for } k=1 \\ \frac{\partial \theta_{p, k-1}}{\partial \tau}+\frac{\partial \theta_{f, k-1}}{\partial \tau}-\frac{\partial \theta_{f, k}}{\partial \tau}, & \text { for } k>1\end{array}, \quad 0 \leq \eta \leq 1\right.$

$\frac{\partial \theta_{p, k}(0, \tau)}{\partial \eta}=0 ; \quad \frac{\partial \theta_{p, k}(1, \tau)}{\partial \eta}+\operatorname{Bi} \theta_{p, k}(1, \tau)=\Delta \gamma_{k}$

where,

$G_{k}(\eta, \tau)=-\left[\frac{\partial \theta_{f, k}}{\partial \tau}+\tau_{r} \frac{\partial^{2} \theta_{f, k}}{\partial \tau^{2}}\right]=-\left[F^{\prime}(\tau)+\tau_{r} F^{\prime \prime}(\tau)\right](1+1 / \mathrm{Bi}-\eta) ;$

$\Delta \gamma_{k}=\gamma(\tau)-\gamma_{k-1}$

An analytical integral transformation of the problem defined by Eqs. (14a-g) is now developed by using the ideas in the generalized integral transform technique (GITT), as described in [20-28]. Follow- ing the basic steps in this integral transform approach, an appropriate eigenvalue problem must be chosen, here taken as:

$\frac{d^{2} \psi_{i}(\eta)}{d \eta^{2}}+\mu_{i}^{2} \psi_{i}(\eta)=0$

$\frac{d \psi_{i}(0)}{d \eta}=0 ; \quad \frac{d \psi_{i}(1)}{d \eta}+B i \psi_{i}(1)=0$

Eq. (16) above are readily solved, to yield the eigenfunctions, transcendental equation for the computation of eigenvalues, orthogonality property and norms, as follows:

$\psi_{i}(\eta)=\cos \left(\mu_{i} \eta\right) ; \quad \mathrm{Bi}=\mu_{i} \tan \left(\mu_{i}\right) ;$

$\int_{0}^{1} \psi_{i}(\eta) \psi_{j}(\eta) d \eta=\left\{\begin{array}{l}0, \mathrm{i} \neq \mathrm{j} \\ \mathrm{N}_{\mathrm{i}}, \mathrm{i}=\mathrm{j}\end{array} ; \quad N_{\mathrm{i}}=\int_{0}^{1} \psi_{i}^{2}(\eta) d \eta=\frac{\mu_{i}^{2}+\mathrm{Bi}^{2}+\mathrm{Bi}}{2\left(\mu_{i}^{2}+\mathrm{Bi}^{2}\right)}\right.$

$(17 \mathrm{c}, \mathrm{d})$

Also, the eigenvalue problem defined by Eq. (16) allows for a definition of the following integral transform pair:

$\bar{\theta}_{k, i}(\tau)=\int_{0}^{1} \tilde{\psi}_{i}(\eta) \theta_{p, k}(\eta, \tau) d \eta, \quad$ transform;

$\theta_{p, k}(\eta, \tau)=\sum_{i=1}^{\infty} \tilde{\psi}_{i}(\eta) \bar{\theta}(\tau), \quad$ inverse

where, $\tilde{\psi}(\eta)=\psi_{\mathrm{i}}(\eta) / N_{\mathrm{i}}^{1 / 2}$ are the normalized eigenfunctions.

To perform the integral transformation, Eq. (14a) and the initial conditions (14b,c) should first be multiplied by the normalized eigenfunction, $\tilde{\psi}(\eta)$, and then integrated over the domain $[0,1]$ in the $\eta$ direction. After employing the inverse formula (18b), and the boundary conditions, Eqs. (14f,g), the following coupled transformed system of second order ordinary differential equations in time is obtained:

$\frac{d^{2} \bar{\theta}_{k, i}(\tau)}{d \tau^{2}}+\frac{1}{\tau_{r}} \frac{d \bar{\theta}_{k, i}(\tau)}{d \tau}+\frac{\mu_{i}^{2}}{\tau_{r}} \bar{\theta}_{k, i}(\tau)=\bar{G}_{k, i}(\tau), \quad \tau_{k-1}<\tau<\tau_{k}$

$\bar{\theta}_{k, i}\left(\tau_{k-1}\right)=\bar{f}_{i} ; \quad \frac{d \bar{\theta}_{k, i}\left(\tau_{k-1}\right)}{d \tau}=\bar{g}_{i}$

where

$\bar{G}_{k, i}(\tau)=\frac{1}{\tau_{r}}\left[\int_{0}^{1} G_{k}(\eta, \tau) \tilde{\psi}_{i}(\eta) d \eta+\tilde{\psi}_{i}(1) \Delta \gamma_{k}\right]$

$\bar{f}_{i}=\left\{\begin{array}{l}\int_{0}^{1}\left(1-\theta_{f, k}\right) \tilde{\psi}_{i}(\eta) d \eta, \text { for } k=1 \\ \int_{0}^{1}\left(\theta_{p, k-1}+\theta_{f, k-1}-\theta_{f, k}\right) \tilde{\psi}_{i}(\eta) d \eta, \text { for } k>1\end{array}\right.$

$(20 \mathrm{a}-\mathrm{c})$

$\bar{g}_{i}=\left\{\begin{array}{l}\int_{0}^{1} \frac{\partial \theta_{f, k}}{\partial \tau} \tilde{\psi}_{i}(\eta) d \eta, \text { for } k=1 \\ \int_{0}^{1}\left(\frac{\partial \theta_{p, k-1}}{\partial \tau}+\frac{\partial \theta_{f, k-1}}{\partial \tau}-\frac{\partial \theta_{f, k}}{\partial \tau}\right) \tilde{\psi}_{i}(\eta) d \eta, \text { for } k>1\end{array}\right.$

The coupling of the transformed potentials is evident in the term $\bar{G}_{\mathrm{k}, \mathrm{i}}(\tau)$, more specifically in the boundary transient term that appears in

Table 1

Comparison of the average temperature for $Q(\tau)=0$ and $\tau_{\mathrm{r}}=10^{-5}$ against the exact solution for $Q(\tau)=0$ and $\tau_{\mathrm{r}}=0$

\begin{tabular}{|c|c|c|c|c|c|}
\hline$\tau$ & $\theta_{\mathrm{av}}(\tau)^{\mathrm{a}}$ & $\theta_{\mathrm{av}}(\tau)^{\mathrm{b}}$ & $\theta_{\mathrm{av}}(\tau)^{\mathrm{c}}$ & $\theta_{\mathrm{av}}(\tau)^{\mathrm{d}}$ & $\theta_{\mathrm{av}}(\tau)^{\mathrm{e}}$ \\
\hline \multicolumn{6}{|c|}{$\mathrm{Bi}=0.1$} \\
\hline 0.01 & 0.9990 & 0.9990 & 0.9990 & 0.9990 & 0.9990 \\
\hline 0.10 & 0.9902 & 0.9902 & 0.9902 & 0.9902 & 0.9902 \\
\hline 1.00 & 0.9076 & 0.9076 & 0.9076 & 0.9075 & 0.9076 \\
\hline \multicolumn{6}{|l|}{$\mathrm{Bi}=1$} \\
\hline 0.01 & 0.9907 & 0.9907 & 0.9908 & 0.9902 & 0.9907 \\
\hline 0.10 & 0.9196 & 0.9196 & 0.9197 & 0.9157 & 0.9196 \\
\hline 1.00 & 0.4704 & 0.4704 & 0.4705 & 0.4680 & 0.4704 \\
\hline
\end{tabular}

a-GITT approach, b-LTM, c-FVGM, d-CIEA-lumped formulation [17], e-exact solution $\left(\tau_{\mathrm{r}}=0\right)$. 
Table 2

Convergence behavior and comparison of the local temperature for a square wave pulse at different dimensionless times and positions $\left(\mathrm{Bi}=0.1\right.$ and $\tau_{\mathrm{r}}=10^{-2}$ and 1$)$

\begin{tabular}{|c|c|c|c|c|c|c|c|}
\hline \multicolumn{8}{|c|}{$Q(\tau)=$ square wave pulse; $\mathrm{Bi}=0.1$ and $\tau_{\mathrm{r}}=10^{-2}$} \\
\hline \multicolumn{8}{|l|}{$\theta$} \\
\hline $\bar{N}$ & 20 & 40 & 60 & 80 & 100 & LTM & FVGM \\
\hline$\tau=1.2$ and $\eta=0.1$ & 1.3096 & 1.3096 & 1.3096 & 1.3096 & 1.3096 & 1.3102 & 1.3096 \\
\hline$\tau=1.2$ and $\eta=0.9$ & 0.92196 & 0.92196 & 0.92196 & 0.92196 & 0.92196 & 0.92240 & 0.92197 \\
\hline$\tau=1.8$ and $\eta=0.1$ & 1.8715 & 1.8715 & 1.8715 & 1.8715 & 1.8715 & 1.8716 & 1.8715 \\
\hline$\tau=1.8$ and $\eta=0.9$ & 1.4185 & 1.4185 & 1.4185 & 1.4185 & 1.4185 & 1.4156 & 1.4185 \\
\hline \multicolumn{8}{|c|}{$Q(\tau)=$ square wave pulse; $\mathrm{Bi}=0.1$ and $\tau_{\mathrm{r}}=1$} \\
\hline \multicolumn{8}{|l|}{$\theta$} \\
\hline $\bar{N}$ & 60 & 80 & 100 & 120 & 140 & LTM & FVGM \\
\hline$\tau=1.2$ and $\eta=0.1$ & 1.0715 & 1.0714 & 1.0714 & 1.0714 & 1.0714 & 1.0713 & 1.0717 \\
\hline$\tau=1.2$ and $\eta=0.9$ & 0.92667 & 0.92664 & 0.92663 & 0.92662 & 0.92662 & 0.92643 & 0.92658 \\
\hline$\tau=1.8$ and $\eta=0.1$ & 1.4935 & 1.4935 & 1.4935 & 1.4935 & 1.4934 & 1.4954 & 1.4934 \\
\hline$\tau=1.8$ and $\eta=0.9$ & 0.89907 & 0.89905 & 0.89904 & 0.89903 & 0.89903 & 0.89750 & 0.89901 \\
\hline
\end{tabular}

$\Delta \gamma_{\mathrm{k}}$. The truncated version of system (19) can be numerically solved with the use of initial value problem solvers with automatic error control, such as in subroutine DIVPAG from the IMSL Library [31]. After the system (19a) is solved for the transformed potentials $\bar{\theta}_{\mathrm{k}, \mathrm{i}}(\tau)$, the temperature field is recovered through the inverse formula (18b) and Eq. (13).

Eq. (21a) defines the average potential $\theta_{\mathrm{av}}(\tau)$, which after introducing both the inverse formula given by Eq. (18b) and Eq. (13), results in Eq. (21b) below:

$\theta_{a v}(\tau)=\int_{0}^{1} \theta(\eta, \tau) \mathrm{d} \eta ; \quad \theta_{a v}(\tau)=F(\tau) \bar{h}_{1, i}+\left[F(\tau)+\gamma_{k-1}\right] \frac{\bar{h}_{2, i}}{\operatorname{Bi}}+\sum_{i=1}^{\infty} \bar{h}_{2, i} \bar{\theta}_{i}(\tau)$

$\bar{h}_{1, i}=\int_{0}^{1}(1-\eta) \tilde{\psi}_{i}(\eta) d \eta ; \quad \bar{h}_{2, i}=\int_{0}^{1} \tilde{\psi}_{i}(\eta) d \eta$

\section{Results and discussion}

Numerical results were computed with different values of Biot number, $\mathrm{Bi}$, dimensionless relaxation time, $\tau_{\mathrm{r}}$, and the two different functional forms for the wave pulse (square and triangular), $Q(\tau)$, at the boundary $\eta=0$. In the GITT solution, the transformed ordinary differential system given by Eq.(19) was verified with the analytical symbolic solution and numerically solved through subroutine DIVPAG of the IMSL Library [31] with a prescribed relative error target of $10^{-6}$ in all computational runs.

The numerical code was also validated with comparisons against the exact integral transform solution for the classical heat conduction problem $\left(\tau_{\mathrm{r}}=0\right)$ and with the approximate formulation obtained with the coupled integral equations approach (CIEA) [32], employing the improved lumped-differential formulation represented by the formulae $\mathrm{H}_{1,1} / \mathrm{H}_{1,1}[17]$. Thus, the present results for the average temperature were compared with those from reference [17], for the situation $Q(\tau)=0$ and $\tau_{\mathrm{r}}=10^{-5}$. One may observe in Table 1 the excellent agreement between the results of the present GITT approach with the exact solution for $Q(\tau)=0$ and $\tau_{\mathrm{r}}=0$ for both Biot numbers considered, $\mathrm{Bi}=0.1$ and 1.0 , as well as with the results from a Laplace transform method with numerical inversion (LTM) and the method of lines variant employing finite volumes for the spatial discretization and Gear's method for the time integration (FVGM). However, when Biot number is increased, the lumped-differential formulation $\mathrm{H}_{1,1} /$ $\mathrm{H}_{1,1}$ given by Reis et al. [17] looses adherence with respect to the other fully local formulations of the hyperbolic heat conduction problem.

Tables 2-5 illustrate the convergence behavior of the eigenfunction expansions in the GITT solution for the two types of wave pulses adopted, as well as the comparison with the results generated with the LTM and FVGM approaches. Tables 2 and 3 show results for the square wave pulse with $\mathrm{Bi}=0.1$ and 1 , respectively, and with dimensionless relaxation times $\tau_{\mathrm{r}}=10^{-2}$ and 1 . The dimensionless times adopted in such analysis are within the range of the thermal perturbation (see Fig. 1) and the dimensionless positions were chosen closer to the boundaries, since it is expected that such representative values are the most relevant ones for the convergence behavior analysis. The results of GITT reach convergence to five significant digits at different rates, for instance, in the case $\mathrm{Bi}=0.1$ and $\tau_{\mathrm{r}}=10^{-2}$, $N=20$ terms are needed to achieve the referred convergence to five digits, while for $\mathrm{Bi}=0.1$ and $\tau_{\mathrm{r}}=1, \mathrm{Bi}=1$ and $\tau_{\mathrm{r}}=10^{-2}$, and $\mathrm{Bi}=1$ and

Table 3

Convergence behavior and comparison of the local temperature for a square wave pulse at different dimensionless times and positions $\left(\mathrm{Bi}=1\right.$ and $\tau_{\mathrm{r}}=10^{-2}$ and 1$)$

\begin{tabular}{|c|c|c|c|c|c|c|c|}
\hline \multicolumn{8}{|c|}{$Q(\tau)=$ square wave pulse; $\mathrm{Bi}=1$ and $\tau_{\mathrm{r}}=10^{-2}$} \\
\hline \multicolumn{8}{|l|}{$\theta$} \\
\hline $\bar{N}$ & 20 & 40 & 60 & 80 & 100 & LTM & FVGM \\
\hline$\tau=1.2$ and $\eta=0.1$ & 0.86638 & 0.86639 & 0.86640 & 0.86640 & 0.86640 & 0.86706 & 0.86641 \\
\hline$\tau=1.2$ and $\eta=0.9$ & 0.37887 & 0.37889 & 0.37889 & 0.37889 & 0.37889 & 0.37971 & 0.37890 \\
\hline$\tau=1.8$ and $\eta=0.1$ & 1.2456 & 1.2456 & 1.2456 & 1.2456 & 1.2456 & 1.2455 & 1.2456 \\
\hline$\tau=1.8$ and $\eta=0.9$ & 0.62947 & 0.62948 & 0.62948 & 0.62948 & 0.62948 & 0.62778 & 0.62949 \\
\hline \multicolumn{8}{|c|}{$Q(\tau)=$ square wave pulse; $\mathrm{Bi}=1$ and $\tau_{\mathrm{r}}=1$} \\
\hline \multicolumn{8}{|l|}{$\theta$} \\
\hline $\bar{N}$ & 60 & 80 & 100 & 120 & 140 & LTM & FVGM \\
\hline$\tau=1.2$ and $\eta=0.1$ & 0.97771 & 0.97771 & 0.97771 & 0.97771 & 0.97771 & 0.97766 & 0.97828 \\
\hline$\tau=1.2$ and $\eta=0.9$ & 0.63790 & 0.63781 & 0.63776 & 0.63772 & 0.63769 & 0.63738 & 0.63753 \\
\hline$\tau=1.8$ and $\eta=0.1$ & 1.1833 & 1.1834 & 1.1834 & 1.1835 & 1.1835 & 1.1856 & 1.1835 \\
\hline$\tau=1.8$ and $\eta=0.9$ & 0.52784 & 0.52779 & 0.52776 & 0.52774 & 0.52773 & 0.52590 & 0.52764 \\
\hline
\end{tabular}


Table 4

Convergence behavior and comparison of the local temperature for a triangular wave pulse at different dimensionless times and positions $\left(\mathrm{Bi}=0.1\right.$ and $\tau_{\mathrm{r}}=10^{-2}$ and 1$)$

\begin{tabular}{|c|c|c|c|c|c|c|c|}
\hline \multicolumn{8}{|c|}{$Q(\tau)=$ triangular wave pulse; $\mathrm{Bi}=0.1$ and $\tau_{\mathrm{r}}=10^{-2}$} \\
\hline \multicolumn{8}{|c|}{$\theta$} \\
\hline $\bar{N}$ & 20 & 40 & 60 & 80 & 100 & LTM & FVGM \\
\hline$\tau=1.2$ and $\eta=0.1$ & 0.95641 & 0.95641 & 0.95641 & 0.95641 & 0.95641 & 0.95610 & 0.95641 \\
\hline$\tau=1.2$ and $\eta=0.9$ & 0.87310 & 0.87310 & 0.87310 & 0.87310 & 0.87310 & 0.87317 & 0.87310 \\
\hline$\tau=1.8$ and $\eta=0.1$ & 1.3445 & 1.3445 & 1.3445 & 1.3445 & 1.3445 & 1.3446 & 1.3445 \\
\hline$\tau=1.8$ and $\eta=0.9$ & 1.0210 & 1.0210 & 1.0210 & 1.0210 & 1.0210 & 1.0227 & 1.0210 \\
\hline \multicolumn{8}{|c|}{$Q(\tau)=$ triangular wave pulse; $\mathrm{Bi}=0.1$ and $\tau_{\mathrm{r}}=1$} \\
\hline \multicolumn{8}{|c|}{ 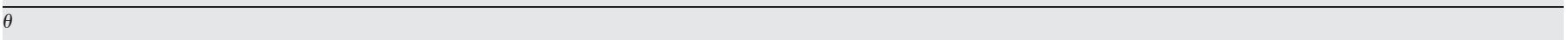 } \\
\hline $\bar{N}$ & 60 & 80 & 100 & 120 & 140 & LTM & FVGM \\
\hline$\tau=1.2$ and $\eta=0.1$ & 1.0762 & 1.0761 & 1.0761 & 1.0761 & 1.0761 & 1.0761 & 1.0764 \\
\hline$\tau=1.2$ and $\eta=0.9$ & 0.92667 & 0.92664 & 0.92663 & 0.92662 & 0.92662 & 0.92621 & 0.92658 \\
\hline$\tau=1.8$ and $\eta=0.1$ & 1.7031 & 1.7031 & 1.7031 & 1.7031 & 1.7031 & 1.7032 & 1.7031 \\
\hline$\tau=1.8$ and $\eta=0.9$ & 0.89907 & 0.89905 & 0.89904 & 0.89903 & 0.89903 & 0.89863 & 0.89901 \\
\hline
\end{tabular}

$\tau_{\mathrm{r}}=1$, the convergence was attained, respectively, with $80 \leq N \leq 100$, $60 \leq N \leq 80$ and $80 \leq N \leq 100$. Tables 2 and 3 also bring a comparison with the LTM and FVGM approaches, and one may notice the excellent agreement among the three sets of results, obtained through fully independent approaches. In general, the GITT and FVMG results are in agreement to four significant digits, while with those of the LTM approach an agreement to three significant digits can be verified. The same observations as above can be extended to Tables 4 and 5 for the case of a triangular wave pulse. It is important to mention that the results of the LTM approach were obtained with a tolerance of $10^{-5}$ in the subroutine DINLAP for numerical inversion of the Laplace transform, IMSL Library [27], while those of the FVGM approach were produced with a mesh of $M=125$ points in the spatial variable discretization.

Figs. 2 and 3 show the time evolution of the temperature distribution for $\mathrm{Bi}=0.1$ and 1 and $\tau_{\mathrm{r}}=1 \times 10^{-2}$ and 1 at the dimensionless times $\tau=0.3,0.9,1.5,2.1,2.7$ and 3.0. From these figures, one may analyze the influence of the Biot number and relaxation time in the thermal wave pulses propagation in the conductive medium. For such square and triangular thermal wave pulses at the boundary $\eta=0$, the effect of the waves thermal propagation is more clearly noticeable. Initially, until the instant $\tau=1$, there is no thermal excitation at $\eta=0$, which occurs in the interval $1 \leq \tau \leq 2$. After the beginning of the heat source excitation, it is observed that the thermal wave propagation in the medium results in a more significant temperature raise for smaller Biot numbers. In the case of $\mathrm{Bi}=0.1$ and $\tau_{\mathrm{r}}=1 \times 10^{-2}$, for times $\tau=0.3$ and 0.9 when there is no thermal excitation at $\eta=0$, the temperature gradient in the medium is still small due the fairly low convection heat exchange rate at $\eta=1$. In the time instant $\tau=1.5$, when the thermal wave pulse is already occurring for a 0.5 dimensionless time interval, dimensionless temperature rises above unity and, due to the small $\tau_{\mathrm{r}}$ value (i.e., high speed of thermal wave propagation in the medium), the thermal wave rapidly increases temperature over the whole region. Still for $\mathrm{Bi}=0.1$, but for $\tau \geq 2.1$, it can be observed that the thermal waves propagation ends up in an approximately uniform temperature field, due to the low dissipation at $\eta=1$.

In the case of $\mathrm{Bi}=0.1$ and $\tau_{\mathrm{r}}=1$ the trends above described are essentially similar but with a slower behavior in time, due to the milder speed of the thermal wave propagation when the relaxation time is smaller. In contrast, more pronounced temperature gradients are then observed. In the case of $\mathrm{Bi}=1$ and $\tau_{\mathrm{r}}=1 \times 10^{-2}$, the convective dissipation at $\eta=1$ is more significant, consequently the temperatures at $\eta=1$ may be lower than at $\eta=0$. However, for $\mathrm{Bi}=1$ and $\tau_{\mathrm{r}}=1$, the thermal waves will reach the position $\eta=1$ at longer times, consequently the temperatures will increase until the thermal waves stop reaching this position. Therefore, for the case of larger Biot number, a similar hyperbolic behavior for both forms of heat pulse has been observed. However, the effect of the energy dissipation at $\eta=1$ becomes more important and competes with the excitation source at $\eta=0$. As expected, the hyperbolic nature becomes more evident for increasing relaxation time, and the temperature distribution further deviates from the classical Fourier modeling behavior.

\section{Conclusions}

Hyperbolic heat conduction in a finite slab, subjected to boundary conditions of prescribed heat flux and convection heat transfer, has been analyzed by employing the generalized integral transform technique (GITT). The proposed approach offers reliable results for the temperature field, as demonstrated in the range of Biot numbers

Table 5

Convergence behavior and comparison of the local temperature for a triangular wave pulse at different dimensionless times and positions $\left(\mathrm{Bi}=1\right.$ and $\tau_{\mathrm{r}}=10^{-2}$ and 1 )

\begin{tabular}{|c|c|c|c|c|c|c|c|}
\hline \multicolumn{8}{|c|}{$Q(\tau)=$ riangular wave pulse; $\mathrm{Bi}=1$ and $\tau_{\mathrm{r}}=10^{-2}$} \\
\hline \multicolumn{8}{|l|}{$\bar{\theta}$} \\
\hline $\bar{N}$ & 20 & 40 & 60 & 80 & 100 & LTM & FVGM \\
\hline$\tau=1.2$ and $\eta=0.1$ & 0.51320 & 0.51322 & 0.51322 & 0.51323 & 0.51323 & 0.52350 & 0.51324 \\
\hline$\tau=1.2$ and $\eta=0.9$ & 0.33468 & 0.33469 & 0.33470 & 0.33470 & 0.33470 & 0.34416 & 0.33471 \\
\hline$\tau=1.8$ and $\eta=0.1$ & 0.77353 & 0.77354 & 0.77355 & 0.77355 & 0.77355 & 0.77243 & 0.77356 \\
\hline$\tau=1.8$ and $\eta=0.9$ & 0.36362 & 0.36363 & 0.36363 & 0.36363 & 0.36363 & 0.36958 & 0.36364 \\
\hline \multicolumn{8}{|c|}{$Q(\tau)=$ triangular wave pulse; $\mathrm{Bi}=1$ and $\tau_{\mathrm{r}}=1$} \\
\hline \multicolumn{8}{|l|}{$\bar{\theta}$} \\
\hline $\bar{N}$ & 60 & 80 & 100 & 120 & 140 & LTM & FVGM \\
\hline$\tau=1.2$ and $\eta=0.1$ & 0.98239 & 0.98239 & 0.98239 & 0.98239 & 0.98239 & 0.98236 & 0.98296 \\
\hline$\tau=1.2$ and $\eta=0.9$ & 0.63790 & 0.63781 & 0.63776 & 0.63772 & 0.63769 & 0.63717 & 0.63753 \\
\hline$\tau=1.8$ and $\eta=0.1$ & 1.3930 & 1.3930 & 1.3931 & 1.3931 & 1.3931 & 1.3933 & 1.3932 \\
\hline$\tau=1.8$ and $\eta=0.9$ & 0.52784 & 0.52779 & 0.52776 & 0.52774 & 0.52773 & 0.52707 & 0.52764 \\
\hline
\end{tabular}




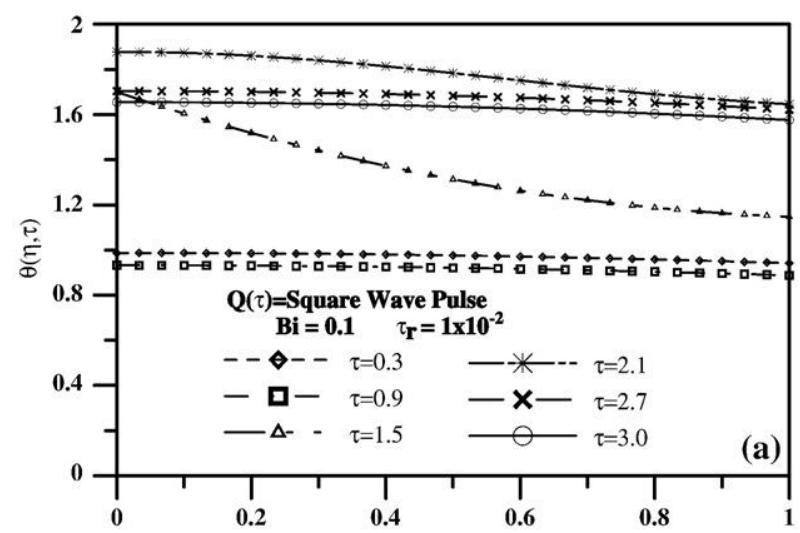

$\eta$
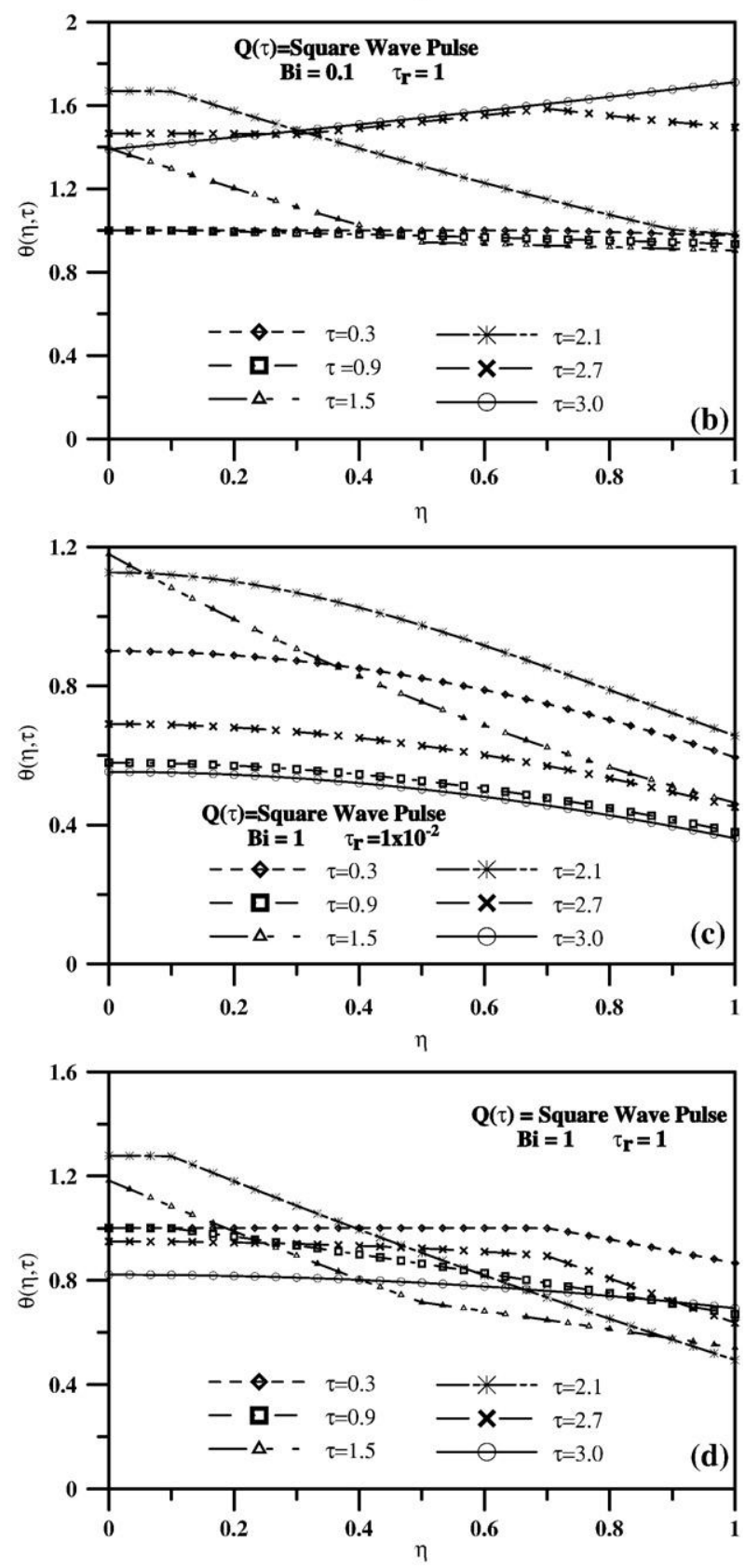

Fig. 2. Evolution of the temperature field for a square wave pulse at different dimensionless times and positions: (a) $\mathrm{Bi}=0.1$ and $\tau_{\mathrm{r}}=10^{-2}$; (b) $\mathrm{Bi}=0.1$ and $\tau_{\mathrm{r}}=1$; (c) $\mathrm{Bi}=1$ and $\tau_{\mathrm{r}}=10^{-2}$; (d) $\mathrm{Bi}=1$ and $\tau_{\mathrm{r}}=1$.
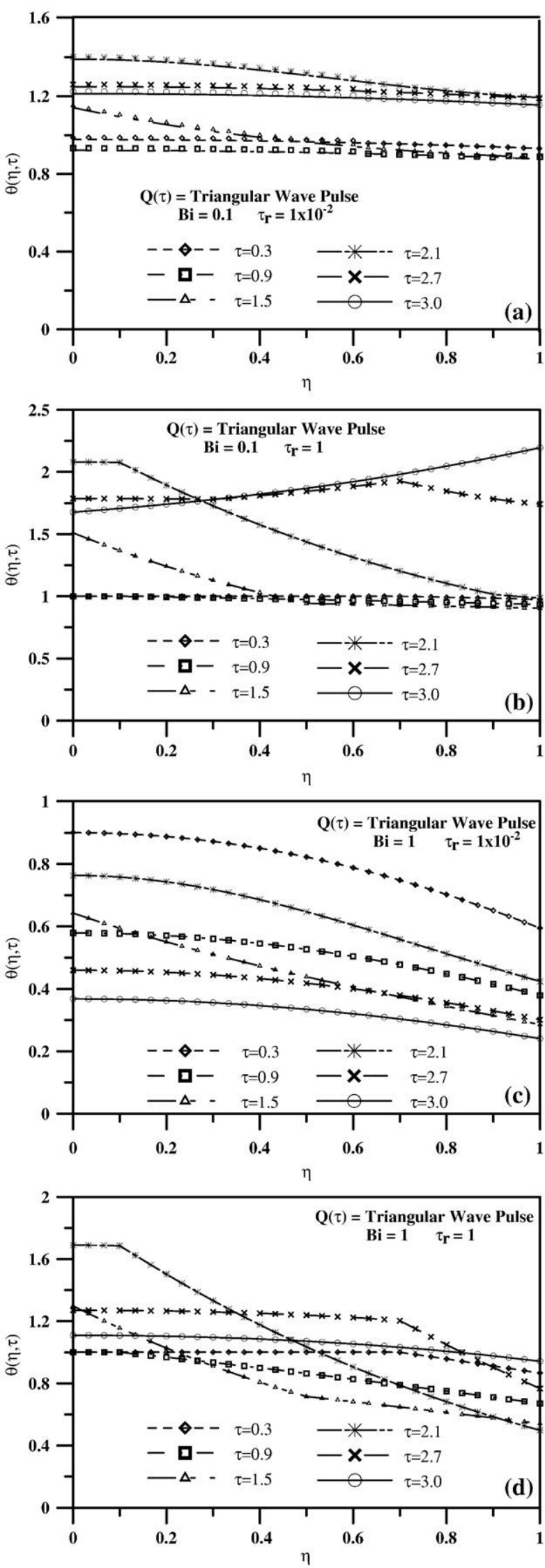

Fig. 3. Evolution of the temperature field for a triangular wave pulse at different dimensionless times and positions: (a) $\mathrm{Bi}=0.1$ and $\tau_{\mathrm{r}}=10^{-2}$; (b) $\mathrm{Bi}=0.1$ and $\tau_{\mathrm{r}}=1$; (c) $\mathrm{Bi}=1$ and $\tau_{\mathrm{r}}=10^{-2}$; (d) $\mathrm{Bi}=1$ and $\tau_{\mathrm{r}}=1$. 
and dimensionless relaxation times analyzed, with good agreement against the results of two alternative and independent approaches based on the Laplace transform with numerical inversion (LTM) and on the method of lines with finite volumes and Gear's algorithm (FVGM). Results for the temperature distributions were produced for square and triangular wave pulses, illustrating the major features of hyperbolic heat transfer as a function of the two governing parameters, Biot number and dimensionless relaxation time.

\section{Acknowledgements}

The authors would like to acknowledge the financial support provided by $\mathrm{CNPq} /$ Brasil.

\section{References}

[1] P. Vernotte, Les paradoxes de la théorie continue de l'équation de la chaleur, Comptes Rendus Hebdomadaires des Séances de l'Académie des Sciences 246 (1958) 3154-3155.

[2] C. Cattaneo, A form of heat conduction equation which eliminates the paradox of instantaneous propagation, Comptes Rendus Hebdomadaires des Séances de l'Académie des Sciences 247 (1958) 431-433.

[3] H.E. Jackson, C.T. Walker, Thermal conductivity, second sound and phononphonon interactions in NaF, Physical Review B 3 (1971) 1428-1439.

[4] V. Narayanamurti, R.C. Dynes, Observation of second sound in bismuth, Physical Review Letters 28 (1972) 1461-1465.

[5] A.M. Brazhnikov, V.A. Karpychev, A.V. Luikov, One engineering method of calculating heat conduction processes, Inhenerno Fizicheskij Zhurnal 28 (1975) 677-679.

[6] S. Sieniutycz, Variational principles of classical type for non-coupled nonstationary irreversible transport processes with convective motion and relaxation, International Journal of Heat and Mass Transfer 20 (1977) 1221-1231.

[7] D.D. Joseph, L. Preziosi, Heat waves, Reviews of Modern Physics 61 (1989) 41-73.

[8] D.D. Joseph, L. Preziosi, Addendum to the paper "Heat waves", Reviews of Modern Physics 62 (1990) 375-391.

[9] W. Kaminski, Hyperbolic heat conduction equation for materials with a nonhomogeneous inner structure, Journal of Heat Transfer 112 (1990) 555-560.

[10] M.N. Özisik, D.Y. Tzou, On the wave theory in heat conduction, Journal of Heat Transfer 116 (1994) 526-535.

[11] H.R.B. Orlande, M.N. Özisik, Simultaneous estimations of thermal diffusivity and relaxation time with a hyperbolic heat conduction model, Proceedings of the Tenth International Heat Transfer Conference, Bringhton UK, vol. 6, 1994, pp. 403-408.

[12] A. Barletta, Hyperbolic propagation of an axisymmetric thermal signal in an infinite solid medium, International Journal of Heat and Mass Transfer 39 (1996) 3261-3271.

[13] A.E. Kronberg, A.H. Benneker, K.R. Westerterp, Notes on wave theory in heat conduction: a new boundary condition, International Journal of Heat and Mass Transfer 41 (1998) 127-137.
[14] A. Barletta, E. Zanchini, Hyperbolic heat conduction and local equilibrium: a second law analysis, International Journal of Heat and Mass Transfer 40 (1997) 1007-1016.

[15] P.J. Antaki, Analysis of hyperbolic heat conduction in a semi-finite slab with surface convection, International Journal of Heat and Mass Transfer 40 (1997) 3247-3250.

[16] S. Liao, General boundary element method for non-linear heat transfer problems governed by hyperbolic heat conduction equation, Computational Mechanics 20 (1997) 397-406

[17] M.C.L. Reis, E.N. Macêdo, J.N.N. Quaresma, Improved lumped-differential formulation in hyperbolic heat conduction, International Communications in Heat and Mass Transfer 27 (2000) 965-974.

[18] L. Wang, Solution structure of hyperbolic heat-conduction equation, International Journal of Heat and Mass Transfer 43 (2000) 365-373.

[19] P. Duhamel, A new finite integral transform pair for hyperbolic conduction problems in heterogeneous media, International Journal of Heat and Mass Transfer 44 (2001) 3307-3320.

[20] R.M. Cotta, Hybrid numerical-analytical approach to nonlinear diffusion problems, Numerical Heat Transfer, Part B-Fundamentals 17 (1990) 217-226.

[21] R.M. Cotta, Integral Transforms in Computational Heat and Fluid Flow, CRC Press, Boca Raton, USA, 1993.

[22] R.M. Cotta, Benchmark results in computational heat and fluid flow: - the integral transform method (invited paper), International Journal of Heat and Mass Transfer 37 (suppl. 1) (1994) 381-393.

[23] R.M. Cotta, The Integral Transform Method in Thermal and Fluid Sciences and Engineering, Begell House, New York, USA, 1998.

[24] L.A. Sphaier, R.M. Cotta, Integral transform analysis of multidimensional eigenvalue problems within irregular domains, Numerical Heat Transfer, Part BFundamentals 38 (2000) 157-175.

[25] L.S.B. Alves, R.M. Cotta, Transient natural convection inside porous cavities: hybrid numerical-analytical solution and mixed symbolic-numerical computation, Numerical Heat Transfer, Part A-Applications 38 (2000) 89-110.

[26] C.A.C. Santos, J.N.N. Quaresma, J.A. Lima, Benchmark Results for Convective Heat Transfer in Ducts: -the Integral Transform Approach, E-Papers, Rio de Janeiro, Brazil, 2001.

[27] R.M. Cotta, M.D. Mikhailov, Hybrid methods and symbolic computations, in: W.J. Minkowycz, E.M. Sparrow, J.Y. Murthy (Eds.), Handbook of Numerical Heat Transfer, 2nd edition, John Wiley, New York, 2006, pp. 493-522, Chapter 16

[28] S.P.A. Paz, E.N. Macêdo, J.N.N. Quaresma, R.M. Cotta, Eigenfunction expansion solution for boundary-layer equations in cylindrical coordinates: simultaneously developing flow in circular tubes, Numerical Heat Transfer, Part A-Applications 52 (2007) 1123-1149.

[29] E.N. Macêdo, R.M. Cotta, H.R.B. Orlande, Local-instantaneous filtering in the integral transform solution of nonlinear diffusion problems, Computational Mechanics 23 (1999) 524-532.

[30] R.R. Gondim, E.N. Macêdo, R.M. Cotta, Hybrid solution for transient internal convection with axial diffusion: integral transforms with local instantaneous filtering, International Journal of Numerical Methods in Heat and Fluid Flow, 17 (2007) 405-417.

[31] IMSL MATH/LIBRARY, User's Manual, Version 2.0, IMSL, Houston, 1991.

[32] R.M. Cotta, M.D. Mikhailov, Heat Conduction: Lumped Analysis, Integral Transforms, Symbolic Computation, Wiley-Interscience, New York, 1997. 